\title{
SOLVENCY REQUIREMENT IN A UNISEX MORTALITY MODEL
}

\author{
BY \\ An Chen, Montserrat Guillen And Elena Vigna
}

\begin{abstract}
Following the EU Gender Directive, that obliges insurance companies to charge the same premium to policyholders of different genders, we address the issue of calculating solvency capital requirements (SCRs) for pure endowments and annuities issued to mixed portfolios. The main theoretical result is that, if the unisex fairness principle is adopted for the unisex premium, the SCR at issuing time of the mixed portfolio calculated with unisex survival probabilities is greater than the sum of the SCRs of the gender-based subportfolios. Numerical results show that for pure endowments the gap between the two is negligible, but for lifetime annuities the gap can be as high as 3-4\%. We also analyze some conservative pricing procedures that deviate from the unisex fairness principle, and find that they lead to SCRs that are lower than the sum of the gender-based SCRs because the policyholders are overcharged at issuing time.
\end{abstract}

\section{KEYWORDS}

SCR, life insurance pricing, unisex tariff, unisex fairness principle, life table, risk margin, Gender Directive, gender discrimination.

JEL codes. C1, C13, C18, C38, J11

\section{INTRODUCTION AND MOTIVATION}

There have been two major changes for insurers operating in the life insurance markets in the European Union since the start of the new millennium.

The first major change is the regulation on gender discrimination, also well known as the EU Gender Directive, see Aseervatham et al. (2016). ${ }^{1}$ This norm establishes that insurance products must be offered at the same price for males and females. Responding to this change, there appears some academic literature addressing the unisex insurance (pricing) practice. For instance, Guillen (2012) indicates that gender information shall be taken into consideration when analyzing the insurance companies' data and risk, despite the ban on the gender 
discrimination on price. Ornelas and Guillen (2013) compare Mexican unisex life tables that are used for insurance purposes with those of the general population. Sass and Seifried (2014) analyze the effects of mandatory unisex tariffs on the optimal insurance demand. Schmeiser et al. (2014) discuss unisex insurance pricing also from the regulator's perspective and Thiery and Van Schoubroeck (2006) deal with the legal aspects of fairness and equality in actuarial risk selection. Chen and Vigna (2017) show how insurance companies can price a portfolio of policies issued to males and females of the same age if they want to respect actuarial fairness at the portfolio level, and they introduce the unisex mortality intensity that is in accordance with the fairness principle.

The second major change in the European context is that capital requirements are now highly regulated with the implementation of the Solvency II directive. The magnitude of the Solvency Capital Requirement (SCR) is of high relevance because it restricts the financial capacity of a company.

In the present paper, we investigate the implications of the adoption of the unisex fairness principle on the SCR, particularly the initial SCR. Taking pure endowments and life annuities as examples, we compute the initial SCR per policy relying on a single "unisex" portfolio and satisfying the initial actuarial fairness principle (Chen and Vigna, 2017), and we compare it with the weighted average of the gender-based per policy SCRs. For these life insurance contracts, we show that the SCR at issuing time of the mixed portfolio calculated with unisex survival probabilities is greater than the sum of the SCRs of the gender-based subportfolios. Moreover, we analyze how the gap between the capital requirements calculated under the two approaches depends on the size of the shock on mortality, on the compositional balance between males and females, and on the type of life insurance products.

An illustration is presented with the mortality experience for the 1950 cohort of males and females in the United Kingdom. The numerical analysis justifies our main theoretical result that the initial SCR relying on a single "unisex" portfolio is higher than the weighted one. However, the difference in the SCR between these two approaches is negligible for pure endowments, and it becomes more substantial for life annuities products. Despite its very stylized nature, our model suggests that insurers should perform internal actuarial analysis with survival tables that distinguish between males and females. To the best of their interests, this leads to a more accurate risk analysis and, interestingly, under some conditions, to a smaller solvency capital compared to the case where information on sex is deleted from their files and no specific analysis by gender-group is done.

In this paper, we also discuss some approaches to computing the unisex tariffs and the SCRs used in practice. Apparently, insurers sometimes deviate from the unisex fairness principle. They either use the price of the riskier gender for all policyholders, or they use a weighted mix of the gender-based survival rates and add an extra loading to it. When such practices are adopted, insurers are overcharging policyholders. Due to the excessive premiums, they need a smaller capital requirement than what the adoption of the unisex fair premium would imply. Some policy-oriented recommendations are given in the conclusions. 
The remainder of the paper is as follows. Section 2 introduces the unisex fairness principle and its implications on the fair premium. Section 3 presents the main theoretical results on the SCR. Section 4 introduces the stochastic mortality model. Section 5 shows numerical applications. Section 6 shows the consequences of some alternative practices. The last section concludes.

\section{UNISEX FAIRNESS PRINCIPLE AND IMPLICATIONS}

In this section, we report the main results in Chen and Vigna (2017), recalling the definition of unisex fairness principle.

Suppose that the insurance company issues a portfolio of identical policies to $m$ males and $n$ females with same age $x$. This portfolio will be called a mixed portfolio in the following. Since the EU Gender Directive, the price to be charged is the same for males and females, say $P^{u}$. Assume that the fair price for the males before the EU Gender Directive was $P^{m}$, calculated according to the males' mortality table

$$
\left[p_{x}^{m}, p_{x+1}^{m}, \ldots, p_{\omega-1}^{m}\right],
$$

where $\omega$ is the maximal allowed age, and that the fair price for the females before the EU Gender Directive was $P^{f}$, calculated according to the females' mortality table

$$
\left[p_{x}^{f}, p_{x+1}^{f}, \ldots, p_{\omega-1}^{f}\right] .
$$

Before the EU Gender Directive, the mixed portfolio consisted of two subportfolios, the first one with $m$ males and price $P^{m}$, the second one with $n$ females and price $P^{f}$. The total amount of premiums collected before the EU Gender Directive was

$$
m \cdot P^{m}+n \cdot P^{f} .
$$

After the EU Gender Directive, in order to respect the actuarial fairness at the global portfolio level, the insurer should collect the amount in (3); therefore,

$$
(m+n) \cdot P^{u}=m \cdot P^{m}+n \cdot P^{f} .
$$

This is formalized in the definition of unisex fairness principle and unisex fair premium.

Definition 2.1 (Unisex fairness principle and unisex fair premium). For a given portfolio of $m$ male policyholders and $n$ female policyholders, whose fair premiums are $P^{m}$ and $P^{f}$ respectively, we say that the unisex tariff $P^{u}$ is calculated according to the unisex fairness principle if

$$
P^{u}=\gamma \cdot P^{m}+(1-\gamma) \cdot P^{f},
$$

where

$$
\gamma=\frac{m}{m+n} \in[0,1] .
$$

In this case, the unisex tariff $P^{u}$ is called unisex fair premium. 
The actuarial fairness for the mixed portfolio can be achieved only by charging the unisex fair premium, whose amount depends only on the fair prices for males and females and on the proportion of each gender in the mixed portfolio. Obviously, when there are no females in the portfolio, $\gamma=1$ and $P^{u}=P^{m}$; when there are no males in the portfolio, $\gamma=0$ and $P^{u}=P^{f}$. Similarly, if $P^{m}=P^{f}$, then $P^{u}=P^{m}=P^{f}$ independent of $\gamma$.

A legitimate question one can have is as follows: How should unisex survival probabilities look like in order to produce a unisex price that is fair? The answer depends on the insurance product issued. We shall address this issue separately for two important life insurance products: pure endowment and lifetime annuity.

Pure endowment The fair prices of a pure endowment insurance contract with a duration $T$ and a unitary payment issued to a male and a female aged $x$ are, respectively,

$$
P^{m}={ }_{T} E_{x}^{m}={ }_{T} p_{x}^{m} e^{-r T} \quad \text { and } \quad P^{f}={ }_{T} E_{x}^{f}={ }_{T} p_{x}^{f} e^{-r T},
$$

where $r$ is the risk-free rate and $e^{-r T}$ is the financial discount factor from $T$ to 0 . According to (4), we have

$$
P^{u}={ }_{T} E_{x}^{u}={ }_{T} p_{x}^{u} e^{-r T},
$$

where

$$
{ }_{T} p_{x}^{u}=\gamma_{T} p_{x}^{m}+(1-\gamma)_{T} p_{x}^{f} .
$$

The interpretation of (6)-(7) is rather important. For the pure endowment, the unisex fair premium is equal to the fair premium issued to a policyholder whose $T$-year survival probability is a weighted average of the $T$-year survival probabilities of males and females, the weights being the proportions of males and females in the portfolio.

Lifetime annuity. The fair prices of a continuous unitary lifetime annuity issued to a male and a female aged $x$ are, respectively, ${ }^{2}$

$$
P^{m}=a_{x}^{m}=\int_{0}^{\omega-x}{ }_{t} p_{x}^{m} e^{-r t} d t \quad \text { and } \quad P^{f}=a_{x}^{f}=\int_{0}^{\omega-x}{ }_{t} p_{x}^{f} e^{-r t} d t .
$$

According to (4), we have

$$
P^{u}=a_{x}^{u}=\int_{0}^{\omega-x}{ }_{t} p_{x}^{u} e^{-r t} d t
$$

where

$$
{ }_{t} p_{x}^{u}=\gamma_{t} p_{x}^{m}+(1-\gamma)_{t} p_{x}^{f}, \quad \text { for all } t \leq \omega-x .
$$

For the lifetime annuity, the unisex fair premium is equal to the fair premium issued to a policyholder whose $t$-year survival probability is a weighted average 
of the $t$-year survival probabilities of males and females for all $t \leq \omega-x$, the weights being the proportions of males and females in the portfolio.

\subsection{Adverse selection issues}

The introduction of the EU Gender Directive can cause the presence of additional adverse selection. We here provide a short discussion of this problem. Sass and Seifried (2014) discuss this problem extensively.

Assume that at time 0, two $x$-aged potential investors (a female and a male) with an amount of $W$, respectively, are interested in investing in the annuity products. Both investors compare the investment in the annuity product with other alternatives in the financial market. Assume both of them are risk averse and have respectively a utility function of $u_{i}(y), i=m, f, u_{i}^{\prime}(y)>0$ and $u_{i}^{\prime \prime}(y)<$ 0 . Assume further that the most attractive alternative in the market provides both investors a utility level of $U_{\text {Investment }}^{i}$.

Assume that two different pension annuity factors (two different prices of continuous unitary annuity payments) are provided to the female and male investors, i.e.,

$$
\bar{a}_{x}^{i}=\int_{0}^{\omega-x}{ }_{t} p_{x}^{i} e^{-r t} d t, \quad i=m, f .
$$

With these prices, the female and male investor will obtain a continuous pension annuity payment of $W / \bar{a}_{x}^{f}$ and $W / \bar{a}_{x}^{m}$, respectively. Assume that in this case, with differentiated pricing (due to the different survival probabilities for male and female) and the resulting payments, both of the investors will invest in the annuity products rather than in the alternative products in the financial market because the annuity product provides a higher utility, i.e.,

$$
U_{\text {Annuity }}^{i}=\int_{0}^{\omega-x}{ }_{t} p_{x}^{i} u_{i}\left(W / \bar{a}_{x}^{i}\right) d t>U_{\text {Investment }}^{i} \quad i=m, f .
$$

If instead the insurance company applies a fair unisex tariff to the annuity products, i.e., in this case, $\bar{a}_{x}^{u}=\int_{0}^{\omega-x} e^{-r t}\left(\frac{1}{2} t p_{x}^{m}+\frac{1}{2} t p_{x}^{f}\right) d t$, then the lifetime utility for the female increases to

$$
\left.U_{\text {Annuity }}^{f}\right|_{\text {unisex }}=\int_{0}^{\omega-x}{ }_{t} p_{x}^{f} u_{f}\left(W / \bar{a}_{x}^{u}\right) d t>U_{\text {Annuity }}^{f}>U_{\text {Investment }}^{f}
$$

due to the increasing utility function and the fact $\bar{a}_{x}^{f}>\bar{a}_{x}^{u}$. In other words, the female still decides for the annuity product. In contrast, the lifetime utility of the male investor becomes now

$$
\left.U_{\text {Annuity }}^{m}\right|_{\text {unisex }}=\int_{0}^{\omega-x}{ }_{t} p_{x}^{m} u_{m}\left(W / \bar{a}_{x}^{u}\right) d t .
$$


As $\bar{a}_{x}^{m}<\bar{a}_{x}^{u}$ and we assume an increasing utility function, it could happen that

$$
\begin{aligned}
& \left.U_{\text {Annuity }}^{m}\right|_{\text {unisex }}=\int_{0}^{\omega-x}{ }_{t}{ }_{t}{ }_{x}^{m} u_{m}\left(W / \bar{a}_{x}^{u}\right) d t \\
& \quad<U_{\text {Investment }}^{m}<\int_{0}^{\omega-x}{ }_{t}{ }_{t} p_{x}^{m} u_{m}\left(W / \bar{a}_{x}^{m}\right) d t=U_{\text {Annuity }}^{m} .
\end{aligned}
$$

If this scenario happens, the good risk (male investor) will decide for the alternative investment instead of investing in the annuity product.

\section{GenERAl RESUlts ON SCR}

\subsection{Standard calculation of SCR}

In order to see how the unisex actuarial fairness impacts on the SCR, we consider both the pure endowment and the lifetime annuity products introduced in the last section.

Generally, the amount of regulatory capital required by Solvency II standards is consistent with a Value-at-Risk assessment at a 99.5\% confidence interval on a 1-year time horizon, see also EIOPA (2014). In Olivieri and Pitacco (2009) and Börger (2010), there are several definitions for the capital charge for the longevity risk. Following them, we choose to define the initial SCR for one single policy as

$$
\operatorname{SCR}^{i}(0)=B E L^{i, s h o c k}(0)-B E L^{i}(0), i=m, f, u,
$$

where $B E L^{i, s h o c k}(0)$ is the best estimate liability value at time 0 under a longevity shock, and $B E L^{i}(0)$ is the best estimate liability value used in the net premium charging. The $S C R^{i}(0)$ measures the buffer that has to be set aside because future benefits could change due to an evolution of mortality experience that is different from that assumed in the calculation of the premium charged. According to Solvency II, insurers are required to assume that a longevity shock will reduce the annual death probabilities by $20 \%$. Returning to the pure endowments and life annuity products considered in the previous section, we obtain for pure endowments:

$$
B E L^{i}(0)=P_{\text {end }}^{i}(0)=S(0, T) e^{-r T}, i=m, f, u,
$$

where $S^{i}(0, T)$ is the survival probability from 0 to $T$ for a policyholder of gender $i$. Note that $S^{i}(0, T)$ is the standard notation for the survival function in continuous-time models: it is the equivalent of ${ }_{T} p_{x}^{i}$ for the survival probability at $T$ for a policyholder aged $x$ used in the previous section. For the continuous 
lifetime annuity products:

$$
B E L^{i}(0)=P_{\text {ann }}^{i}(0)=\int_{0}^{\omega-x} S^{i}(0, s) e^{-r s} d s, i=m, f, u,
$$

where $S^{i}(0, s)$ is the survival probability from 0 to $s$ for a policyholder of gender $i$.

In the following, we need to find a way of introducing the longevity shock in order to obtain the shocked survival probabilities $S^{i, s h o c k}(0, T), i=m, f, u$, where the superscript shock stands for longevity shock. Hereby, we follow the approach of Lin and Cox (2005) and, for a given cohort $x$, the survival probabilities (for all $t>0$ ) are simultaneously shocked. More specifically, we assume

$$
S^{i, \text { shock }}(0, T)=\left(S^{i}(0, T)\right)^{1-\epsilon}, \quad i=m, f, u
$$

where $\epsilon \in[0,1]$ is a constant.

3.1.1. SCR for pure endowment and lifetime annuity. Applying the definition of the SCR per policy in (10) and the shocked survival probability as in (13) to the pure endowment, we obtain

$$
\begin{aligned}
\operatorname{SCR}_{\text {end }}^{i}(\epsilon, 0, T) & =\left[S^{i \text { shock }}(0, T)-S(0, T)\right] e^{-r T} \\
& =\left[\left(S^{i}(0, T)\right)^{1-\epsilon}-S^{i}(0, T)\right] e^{-r T}, i=m, f, u .
\end{aligned}
$$

Applying the definition of the SCR in (10) and the shocked survival probability as in (13) to the annuity, we obtain

$$
\begin{aligned}
\operatorname{SCR}_{\mathrm{ann}}^{i}(\epsilon, 0) & =\int_{0}^{\omega-x}\left[S^{i, s h o c k}(0, s)-S^{i}(0, s)\right] e^{-r s} d s \\
& =\int_{0}^{\omega-x}\left[\left(S^{i}(0, s)\right)^{1-\epsilon}-S^{i}(0, s)\right] e^{-r s} d s, i=m, f, u .
\end{aligned}
$$

We notice from (14) that, for a fixed interest rate $r>0$, the initial SCR at time 0 for the pure endowment with duration $T$ is a function of two variables: the shock $\epsilon \in[0,1]$, and the duration $T$. Similarly, we see from (15) that the initial SCR at time 0 for the lifetime annuity is a function of the shock $\epsilon \in[0,1]$. In the following, we will need to use the dependence of SCR on the different variables, and it is therefore important to highlight it. However, the complete notation with the dependence of $S C R(\cdot)$ on two variables is heavy and often unnecessary. For notational convenience, in the rest of the paper, we will sometimes adopt the 
following simplified notations:

$$
\begin{aligned}
S C R_{\mathrm{end}-T}^{i}(\epsilon) & :=S C R_{\mathrm{end}}^{i}(\epsilon, 0, T) \\
S C R_{\mathrm{ann}}^{i}(\epsilon) & :=S C R_{\mathrm{ann}}^{i}(\epsilon, 0) \\
S C R_{\mathrm{end}}^{i}(\epsilon, \tau) & :=S C R_{\mathrm{end}}^{i}(\epsilon, 0, \tau)
\end{aligned}
$$

In other words, we suppress 0 from the arguments of $S C R(\cdot)$ and leave $S C R(\cdot)$ as a function of $\epsilon$ (and possibly $\tau$ ) only, see (16)-(18); when the duration of the pure endowment $T$ does not change, we just report it in the subscript, see (16); when the duration of the pure endowment $\tau$ does change, we leave it as an argument of $S C R(\cdot)$, see (18).

\subsection{General results}

This section is the mathematical core of the paper. We prove that for the pure endowment and the annuity the adoption of the unisex fairness principle implies that the SCR at issuing time of the mixed portfolio calculated with the unisex survival probability is greater than or equal to the weighted sum of the SCRs of the two gender-based subportfolios. Here, we consider the SCR as calculated at time 0; accordingly, and following the notation of Section 3.1.1, the SCR is modeled as a function of $\epsilon$ (and possibly $\tau$ ) only.

Proposition 3.1. Assume that a portfolio of $m+n$ pure endowments with duration $T$ and sum assured $M=1$ issued to $m$ males and $n$ females aged $x$ is priced according to the unisex fairness principle. Assume that the SCR at time 0 is calculated according to

$$
S C R_{e n d-T}^{i}(\epsilon)=e^{-r T}\left[\left(S_{x}^{i}(T)\right)^{1-\epsilon}-S_{x}^{i}(T)\right] \quad \text { for } \quad i=u, m, f,
$$

where $S_{x}^{i}(T)$ is the $T$-year pre-shock survival probability for age $x$ and gender $i=$ $u, m, f$, and $\epsilon \in[0,1]$. Then, the $S C R$ at time 0 for a unisex policyholder is greater than or equal to the weighted average of the SCRs at time 0 for male and female:

$$
S C R_{\text {end }-T}^{u}(\epsilon) \geq \gamma S C R_{\text {end }-T}^{m}(\epsilon)+(1-\gamma) S C R_{\text {end }-T}^{f}(\epsilon) \quad \forall \epsilon \in[0,1]
$$

where

$$
\gamma=\frac{m}{m+n} \in[0,1]
$$

is the proportion of males in the portfolio. Assuming that $S_{x}^{m}(T) \neq S_{x}^{f}(T)$, then the inequality in (20) is strict if and only if $\epsilon \in(0,1)$ and $\gamma \in(0,1)$.

Proof. Let us define the function $h(\cdot)$ :

$$
h(\epsilon)=S C R_{\text {end }-T}^{u}(\epsilon)-\left(\gamma S C R_{\text {end }-T}^{m}(\epsilon)+(1-\gamma) S C R_{\text {end }-T}^{f}(\epsilon)\right) .
$$


Claim (20) is equivalent to the non-negativity of the function $h(\epsilon)$. From (19) and (21) (for notational convenience, in the following we will write $S^{i}$ in the place of $\left.S_{x}^{i}(T)\right)$, we have

$h(\epsilon) e^{r T}=\left(S^{u}\right)^{1-\epsilon}-S^{u}-\left(\gamma\left(S^{m}\right)^{1-\epsilon}-\gamma S^{n}+(1-\gamma)\left(S^{f}\right)^{1-\epsilon}-(1-\gamma) S^{f}\right)$.

Due to the unisex fairness principle, the relationship (7) holds true:

$$
S^{u}=\gamma S^{m}+(1-\gamma) S^{f} .
$$

By simplifying, we have

$$
h(\epsilon) e^{r T}=\left(S^{u}\right)^{1-\epsilon}-\left(\gamma\left(S^{m}\right)^{1-\epsilon}+(1-\gamma)\left(S^{f}\right)^{1-\epsilon}\right) .
$$

Let us define the function $f(\cdot)$ :

$$
f(x)=x^{1-\epsilon} .
$$

Then, due to (22), (23) becomes

$$
h(\epsilon) e^{r T}=f\left(\gamma S^{n}+(1-\gamma) S^{f}\right)-\gamma f\left(S^{n}\right)-(1-\gamma) f\left(S^{f}\right) \geq 0,
$$

where the inequality results from applying the Jensen's inequality to the concave function $f(x)$ for $\epsilon \in[0,1]$. Hence, the claim (20) is proven. If $\gamma=0$ (or $\gamma=$ 1) there are no males (or females) in the mixed portfolio, and the inequality becomes an equality. If $\epsilon=0$, then $S C R_{\text {end-T }}^{i}=0$ for all $i=u, m, f$ and the equality holds; if $\epsilon=1$, the function $f(x)$ is linear and the equality holds. If $\epsilon \in(0,1)$ and $\gamma \in(0,1)$, then the function $f(x)$ is strictly concave and the inequality in (20) is strict.

The same result holds as a corollary also for the annuity case. In the following corollary, we use the fact that the annuity is the union of pure endowments with different durations, and adopt the notation introduced in (18).

Corollary 3.2. Assume that a portfolio of $m+n$ lifetime annuities with a unitary payment issued to $m$ males and $n$ females aged $x$ is priced according to the unisex fairness principle. Assume that the SCR at time 0 is calculated according to

$$
\begin{aligned}
S C R_{\text {ann }}^{i}(\epsilon) & =\int_{0}^{\omega-x}\left[\left(S_{x}^{i}(\tau)\right)^{1-\epsilon}-S_{x}^{i}(\tau)\right] e^{-r \tau} d \tau \\
& =\int_{0}^{\omega-x} S C R_{\text {end }}^{i}(\epsilon ; \tau) d \tau \quad \text { for } \quad i=u, m, f,
\end{aligned}
$$

where $S_{x}^{i}(t)$ is the $t$-year pre-shock survival probability for age $x$ and gender $i=$ $u, m, f, S C R_{\text {end }}^{i}(\epsilon ; \tau)$ is the SCR at time 0 for a pure endowment duration $\tau$, and $\epsilon \in[0,1]$. Then, the SCR at time 0 for a unisex policyholder is greater than or 
equal to the volume-related weighted average of the SCRs at time 0 for male and female:

$$
S C R_{a n n}^{u}(\epsilon) \geq \gamma \operatorname{SCR} R_{a n n}^{m}(\epsilon)+(1-\gamma) S C R_{a n n}^{f}(\epsilon) \quad \forall \epsilon \in[0,1]
$$

where

$$
\gamma=\frac{m}{m+n} \in[0,1]
$$

is the proportion of males in the portfolio. Assuming that $S_{x}^{m}(\tau) \neq S_{x}^{f}(\tau)$ for all $\tau$, then the inequality in (25) is strict if and only if $\epsilon \in(0,1)$ and $\gamma \in(0,1)$.

In the corollary, we set $c(t)=1$ for simplicity, but the extension to the general payment $c(t)$ is straightforward.

Proof. Using (24), we have

$$
\begin{gathered}
S C R_{\mathrm{ann}}^{u}(\epsilon)-\left(\gamma S C R_{\mathrm{ann}}^{m}(\epsilon)+(1-\gamma) S C R_{\mathrm{ann}}^{f}(\epsilon)\right)= \\
\int_{0}^{\omega-x}\left[S C R_{\mathrm{end}}^{u}(\epsilon ; \tau)-\gamma S C R_{\mathrm{end}}^{m}(\epsilon ; \tau)-(1-\gamma) S C R_{\mathrm{end}}^{f}(\epsilon ; \tau)\right] d \tau \geq 0,
\end{gathered}
$$

where the inequality is due to the fact that, by Proposition 3.1, the integrand function is positive. The other claims follow easily.

Remark 1. Notice that the results of Proposition 3.1 and Corollary 3.2 hold only at time 0, or issuing time. In general, they do not hold at time $t>0$. Indeed, the unisex fairness principle holds only at issuing time. The reason behind it is that we have used the initial unisex fairness principle in our derivation. This fairness principle can be violated in future time $t>0$.

Remark 2. Proposition 3.1 and Corollary 3.2 remark the impact of the EU Gender Directive on the value of the SCR at issuing time. Indeed, before the EU Gender Directive, the SCR was calculated separately on the two subportfolios and the aggregate $S C R$ was

$$
m S C R^{m}+n S C R^{f}=(m+n) S C R^{\text {weighted }},
$$

where

$$
S C R^{\text {weighted }}=\gamma S C R^{m}+(1-\gamma) S C R^{f} .
$$

After the EU Gender Directive, the insurer can still calculate the SCR in the old way with the two subportfolios. But if, instead, he prefers to calculate the SCR considering a single mixed portfolio with $m+n$ unisex policyholders (maybe because of fiscal incentives), ${ }^{3}$ he should calculate $(m+n) S C R^{u}$. Proposition 3.1 and Corollary 3.2 compare the SCR at issuing time calculated with the two different procedures.

In Section 4, we introduce a stochastic mortality model, and in Section 5, we calibrate it and calculate the difference between the unisex SCR, $S C R^{u}$, and the weighted sum of the gender-based SCRs as in (28). This illustrates the practical implications of the results just proven. 


\section{THE STOCHASTIC MORTALITY MODEL}

In this section, we review the stochastic unisex mortality model introduced in Chen and Vigna (2017). Let us introduce a complete filtered probability space $(\Omega, \mathfrak{F}, \mathbb{P})$ and a filtration $\mathcal{F}_{t}$ of sub- $\sigma$-algebras representing the state of information at time $0 \leq t \leq T$. An insurance company manages a mixed portfolio with $m$ male policyholders and $n$ female policyholders with the same age $x$. For notational convenience, throughout this section, we omit the dependence on $x$ of the mortality processes. We describe the stochastic force of mortality of each gender as an affine process $\lambda$. In other words, the time of death is modeled as the first jump time of a doubly stochastic process with intensity $\lambda$ (see Duffie et al., 2000; Milevsky and Promislow, 2001; Dahl, 2004; Biffis, 2005; Luciano and Vigna, 2008). In particular, the stochastic mortality intensity $\lambda^{m}$ of males and the stochastic mortality intensity $\lambda^{f}$ of females are described by two different Ornstein-Uhlenbeck processes (OU processes) with positive drift and no mean reversion:

$$
\begin{aligned}
& d \lambda^{m}(t)=\mu_{m} \lambda^{m}(t) d t+\sigma_{m} d W^{m}(t), \\
& d \lambda^{f}(t)=\mu_{f} \lambda^{f}(t) d t+\sigma_{f} d W^{f}(t),
\end{aligned}
$$

where $\mu_{i}>0$ and $\sigma_{i}>0$ for $i=m, f$, and $W_{m}$ and $W_{f}$ are two standard Brownian motions under the real-world measure $\mathbb{P}$, correlated with a correlation coefficient $\rho$. The OU process for the mortality intensity is a natural stochastic generalization of the Gompertz law for the force of mortality and is introduced by Luciano and Vigna (2008) where the conditions for its biological reasonableness are also analysed. The survival probability function of males and females can be expressed in closed-form (see, Luciano and Vigna, 2008):

$$
\begin{aligned}
S^{i}(t, T) & =E\left[\exp \left\{-\int_{t}^{T} \lambda^{i}(u) d u\right\} \mid \mathcal{F}_{t}\right]=\exp \left\{\alpha_{i}(\tau)+\beta_{i}(\tau) \lambda^{i}(t)\right\}, i=f, m \\
\alpha_{i}(\tau) & =\frac{\sigma_{i}^{2}}{2 \mu_{i}^{2}} \tau-\frac{\sigma_{i}^{2}}{\mu_{i}^{3}} e^{\mu_{i} \tau}+\frac{\sigma_{i}^{2}}{4 \mu_{i}^{3}} e^{2 \mu_{i} \tau}+\frac{3 \sigma_{i}^{2}}{4 \mu_{i}^{3}}, i=f, m \\
\beta_{i}(\tau) & =\frac{1}{\mu_{i}}\left(1-e^{\mu_{i} \tau}\right), i=f, m
\end{aligned}
$$

where $\tau:=T-t$.

Chen and Vigna (2017) model the mortality intensity of a representative unisex policyholder of the mixed portfolio as a weighted average of the males' and females' mortality intensities, and provide the following definition.

Definition 4.1 (Unisex mortality intensity). For a mixed portfolio of male and female policyholders, whose stochastic mortality intensities are $\lambda^{m}$ and $\lambda^{f}$, respectively, we define the $\xi$-driven unisex mortality intensity by mixing the male and 
female intensities with the weight $\xi \in[0,1]$ :

$$
\lambda_{\xi}^{u}(t)=\xi \lambda^{m}(t)+(1-\xi) \lambda^{f}(t) .
$$

Chen and Vigna (2017) find the survival probability of a unisex policyholder whose mortality intensity is given by (31) (for simplicity, in the following the subscript $\xi$ in the functions $\alpha, \beta_{1}$ and $\beta_{2}$ is omitted).

Proposition 4.2. Conditional on $t$, the survival probability for the remaining time $\tau=T-t$ related to the mixed mortality intensity $\lambda_{\xi}^{u}$ in (31) is given by

$$
\begin{aligned}
S^{u}(t, T) & =E\left[\exp \left\{-\int_{t}^{T} \lambda_{\xi}^{u}(s) d s\right\} \mid \mathcal{F}_{t}\right], \\
& =E\left[\exp \left\{-\int_{t}^{T} \xi \lambda^{m}(s) d s-\int_{t}^{T}(1-\xi) \lambda^{f}(s) d s\right\} \mid \mathcal{F}_{t}\right], \\
& =\exp \left\{\alpha_{u}(\tau)+\beta_{1, u}(\tau) \lambda^{m}(t)+\beta_{2, u}(\tau) \lambda^{f}(t)\right\},
\end{aligned}
$$

with

$$
\begin{gathered}
\beta_{1, u}(\tau)=\frac{\xi}{\mu_{m}}\left(1-e^{\mu_{m} \tau}\right), \\
\beta_{2, u}(\tau)=\frac{1-\xi}{\mu_{f}}\left(1-e^{\mu_{f} \tau}\right),
\end{gathered}
$$

and

$$
\begin{aligned}
\alpha_{u}(\tau)= & \frac{\sigma_{m}^{2} \xi^{2}}{4 \mu_{m}^{3}}\left[\left(e^{\mu_{m} \tau}-2\right)^{2}+2 \mu_{m} \tau-1\right] \\
& +\frac{\sigma_{f}^{2}(\xi-1)^{2}}{4 \mu_{f}^{3}}\left[\left(e^{\mu_{f} \tau}-2\right)^{2}+2 \mu_{f} \tau-1\right] \\
& -\frac{\rho \sigma_{m} \sigma_{f} \xi(\xi-1)}{\mu_{m}^{2} \mu_{f}^{2}\left(\mu_{m}+\mu_{f}\right)}\left\{\mu_{m}^{2}\left(1-e^{\mu_{f} \tau}\right)+\mu_{f}^{2}\left(1-e^{\mu_{m} \tau}\right)\right. \\
& \left.+\mu_{m} \mu_{f}\left[\left(1-e^{\mu_{m} \tau}\right)\left(1-e^{\mu_{f} \tau}\right)+\left(\mu_{m}+\mu_{f}\right) \tau\right]\right\}
\end{aligned}
$$

Proof. Proof can be found in Chen and Vigna (2017).

Finally, among the infinitely many possible weights $\xi \in[0,1]$ of the family (31), Chen and Vigna (2017) identify the critical weight $\xi^{*}$ that generates the fair unisex premium. Noting that the fair premium of a life insurance product is a function $\Pi(\cdot)$ of the mortality intensity of the insured:

$$
P^{i}=\Pi\left(\lambda^{i}\right) \quad \text { for } i=u, m, f,
$$


the identification of the correct weight $\xi^{*}$ can be formalized by the following definition.

Definition 4.3 (Fair unisex mortality intensity). For a given portfolio of $m$ male policyholders and $n$ female policyholders, whose fair gender-based premiums are $P^{m}=\Pi\left(\lambda^{m}\right)$ and $P^{f}=\Pi\left(\lambda^{f}\right)$, respectively, we say that $\lambda_{\xi^{*}}^{u}$ is a fair unisex mortality intensity if the corresponding unisex premium,

$$
P^{u}=\Pi\left(\lambda_{\xi^{*}}^{u}\right)=\Pi\left(\xi^{*} \lambda^{m}+\left(1-\xi^{*}\right) \lambda^{f}\right),
$$

is fair, i.e., it satisfies the unisex fairness principle (4):

$$
\Pi\left(\xi^{*} \lambda^{m}+\left(1-\xi^{*}\right) \lambda^{f}\right)=\gamma \cdot \Pi\left(\lambda^{m}\right)+(1-\gamma) \cdot \Pi\left(\lambda^{f}\right),
$$

where $\gamma=m /(m+n)$, and $\xi^{*}$ is called the fair mortality mixing parameter.

\section{NUMERICAL APPLICATION}

\subsection{Calibration of UK cohort born in 1950}

In this section, we calibrate the mortality model presented in Section 4 and, in the presence of the unisex fairness principle, we investigate the magnitude of the gap between the SCR calculated with the fair unisex mortality intensity and the weighted average of the SCRs of the two subportfolios of males and females. For the calibration of the gender-based mortality intensities, we take data from the Human Mortality Database (HMD hereafter) University of California, Berkeley (USA) and Max Planck Institute for Demographic Research (German) (2002) (data downloaded on: May 6, 2013) and consider the males and females born in 1950 in UK, initial age 35. We adopt the same calibration procedure used in Chen and Vigna (2017) and review the calibration procedure shortly.

For the cohort of initial age $x=35$ and each gender $i=m, f$, we have extrapolated from the HMD 20 observed survival probabilities ${ }_{t} \hat{p}_{x}^{i}, t=1, \ldots, 20$; then, we have calibrated the values of the parameters $\mu_{m}, \mu_{f}, \sigma_{m}, \sigma_{f}$ that appear in the theoretical survival functions $S_{x}^{m}(0, t)$ and $S_{x}^{f}(0, t)$ given by (30) by minimizing the following mean square error:

$$
\frac{1}{20} \sum_{t=1}^{20}\left({ }_{t} \hat{p}_{x}^{i}-S_{x}^{i}(0, t)\right)^{2}
$$

for $i=m, f$. In all cases, the value of the initial observed intensity $\lambda_{x}^{i}(0)$ is set equal to $-\ln \hat{p}_{x}^{i}$. Table 1 reports the calibrated values of the parameters for the male and female generations 1950. 
TABLE 1

CALIBRATED VALUES AND ERRORS FOR MALES AND FEMALES OF COHORT 1950 (INITIAL AGE 35).

\begin{tabular}{lcc}
\hline \hline & Female & Male \\
\hline$\lambda_{x}(0)$ & 0.00075028 & 0.00112463 \\
$\mu_{x}$ & 0.08001563 & 0.08171875 \\
$\sigma_{x}$ & 0.00010305 & 0.00011789 \\
Calibration Error & 0.00000006 & 0.00000007 \\
\hline \hline
\end{tabular}

TABLE 2

FAIR $\xi^{*}$ FOR PURE ENDOWMENT (PE) AND LIFETIME ANNUITY WITH PARAMETERS: $\rho=0.95, r=0.03$, GENERATION BORN IN 1950, INITIAL AGE 35 AND MAXIMAL ALLOWED AGE $\omega=110$.

\begin{tabular}{lccc}
\hline \hline$\gamma=\frac{m}{m+n}$ & PE, $T=20$ & PE, $T=30$ & Lifetime Annuity \\
\hline 0.10 & 0.0991 & 0.0976 & 0.0836 \\
0.25 & 0.2481 & 0.2445 & 0.2154 \\
0.50 & 0.4974 & 0.4932 & 0.4527 \\
0.75 & 0.7481 & 0.7449 & 0.7137 \\
0.90 & 0.8991 & 0.8975 & 0.8823 \\
\hline \hline
\end{tabular}

\subsection{Fair $\xi^{*}$ and SCR}

In this section, we focus on three products (i) pure endowment 20 years, (ii) pure endowment 30 years and (iii) lifetime annuity, which are sold to males and females. We assume that the age at inception of the policy is 35 for the pure endowments and 65 for the annuity. Assuming an interest rate $r=0.03$ and $\rho=0.95,{ }^{4}$ we have calculated $\xi^{*}$ with portfolio gender composition $\gamma$ ranging from $\gamma=0.10$ to $\gamma=0.90$. The fair $\xi^{*}$ values are reported in Table 2 .

Then, we have calculated the unisex SCR and the weighted average of SCRs of males and females for all the products with a variety of shocks $\epsilon$ and a variety of portfolio gender compositions $\gamma$. In particular, using the notation of Section 3.1.1, for

- products $z=$ end-20, end-30, ann,

- shocks $\epsilon=0,0.1,0.2,0.3,0.4,0.5,0.6,0.7,0.8,0.9,1$,

- portfolio gender-compositions $\gamma=0,0.1,0.25,0.5,0.75,0.9,1$,

we have calculated:

- $S C R_{z}^{u}(\epsilon)$,

- $S C R_{z}^{\text {weighted }}(\epsilon)=\gamma \operatorname{SCR} R_{z}^{m}(\epsilon)+(1-\gamma) S C R_{z}^{f}(\epsilon)$,

- their absolute difference $S C R_{z}^{u}(\epsilon)-S C R_{z}^{\text {weighted }}(\epsilon)$,

- their relative difference $\left(S C R_{z}^{u}(\epsilon)-S C R_{z}^{\text {weighted }}(\epsilon)\right) / S C R_{z}^{u}(\epsilon)$.

The main results are displayed in Tables 3 and 4 . 
TABLE 3

SCR ESTIMATION FOR UNISEX $S C R_{z}^{u}(\epsilon)$ (LEFT) AND WEIGHTED SUBPORTFOLIOS $S C R_{z}^{\text {weighted }}(\epsilon)$ (RIGHT), FOR VARYING PROPORTION $(\gamma)$ MALE/FEMALE AND SHOCK $(\epsilon)$.

\begin{tabular}{|c|c|c|c|c|c|c|c|c|c|c|c|c|c|c|c|}
\hline \multicolumn{4}{|c|}{ Pure-endowment 20 years } & \multicolumn{4}{|c|}{$\gamma$} & \multirow[b]{2}{*}{$\epsilon$} & \multicolumn{7}{|c|}{$\gamma$} \\
\hline$\epsilon$ & $0 \%$ & $10 \%$ & $25 \%$ & $50 \%$ & $75 \%$ & $90 \%$ & $100 \%$ & & $0 \%$ & $10 \%$ & $25 \%$ & $50 \%$ & $75 \%$ & $90 \%$ & $100 \%$ \\
\hline 0.1 & 0.002 & 0.002 & 0.002 & 0.002 & 0.003 & 0.003 & 0.003 & 0.1 & 0.002 & 0.002 & 0.002 & 0.002 & 0.003 & 0.003 & 0.003 \\
\hline 0.2 & 0.004 & 0.004 & 0.004 & 0.005 & 0.005 & 0.006 & 0.006 & 0.2 & 0.004 & 0.004 & 0.004 & 0.005 & 0.005 & 0.006 & 0.006 \\
\hline 0.3 & 0.006 & 0.006 & 0.007 & 0.007 & 0.008 & 0.009 & 0.009 & 0.3 & 0.006 & 0.006 & 0.007 & 0.007 & 0.008 & 0.009 & 0.009 \\
\hline 0.4 & 0.008 & 0.008 & 0.009 & 0.010 & 0.011 & 0.011 & 0.012 & 0.4 & 0.008 & 0.008 & 0.009 & 0.010 & 0.011 & 0.011 & 0.012 \\
\hline 0.5 & 0.010 & 0.010 & 0.011 & 0.012 & 0.014 & 0.014 & 0.015 & 0.5 & 0.010 & 0.010 & 0.011 & 0.012 & 0.014 & 0.014 & 0.015 \\
\hline 0.6 & 0.012 & 0.012 & 0.013 & 0.015 & 0.016 & 0.017 & 0.018 & 0.6 & 0.012 & 0.012 & 0.013 & 0.015 & 0.016 & 0.017 & 0.018 \\
\hline 0.7 & 0.014 & 0.015 & 0.016 & 0.017 & 0.019 & 0.020 & 0.021 & 0.7 & 0.014 & 0.015 & 0.016 & 0.017 & 0.019 & 0.020 & 0.021 \\
\hline 0.8 & 0.016 & 0.017 & 0.018 & 0.020 & 0.022 & 0.023 & 0.024 & 0.8 & 0.016 & 0.017 & 0.018 & 0.020 & 0.022 & 0.023 & 0.024 \\
\hline 0.9 & 0.018 & 0.019 & 0.020 & 0.023 & 0.025 & 0.026 & 0.027 & 0.9 & 0.018 & 0.019 & 0.020 & 0.023 & 0.025 & 0.026 & 0.027 \\
\hline 1.0 & 0.020 & 0.021 & 0.023 & 0.025 & 0.028 & 0.029 & 0.030 & 1.0 & 0.020 & 0.021 & 0.023 & 0.025 & 0.028 & 0.029 & 0.030 \\
\hline \multicolumn{4}{|c|}{ Pure-endowment 30 years } & \multicolumn{4}{|c|}{$\gamma$} & & \multicolumn{7}{|c|}{$\gamma$} \\
\hline$\epsilon$ & $0 \%$ & $10 \%$ & $25 \%$ & $50 \%$ & $75 \%$ & $90 \%$ & $100 \%$ & $\epsilon$ & $0 \%$ & $10 \%$ & $25 \%$ & $50 \%$ & $75 \%$ & $90 \%$ & $100 \%$ \\
\hline 0.1 & 0.003 & 0.004 & 0.004 & 0.004 & 0.005 & 0.005 & 0.005 & 0.1 & 0.003 & 0.004 & 0.004 & 0.004 & 0.005 & 0.005 & 0.005 \\
\hline 0.2 & 0.007 & 0.007 & 0.008 & 0.009 & 0.010 & 0.010 & 0.010 & 0.2 & 0.007 & 0.007 & 0.008 & 0.009 & 0.010 & 0.010 & 0.010 \\
\hline 0.3 & 0.011 & 0.011 & 0.012 & 0.013 & 0.014 & 0.015 & 0.016 & 0.3 & 0.011 & 0.011 & 0.012 & 0.013 & 0.014 & 0.015 & 0.016 \\
\hline 0.4 & 0.014 & 0.015 & 0.016 & 0.018 & 0.019 & 0.020 & 0.021 & 0.4 & 0.014 & 0.015 & 0.016 & 0.018 & 0.019 & 0.020 & 0.021 \\
\hline 0.5 & 0.018 & 0.019 & 0.020 & 0.022 & 0.024 & 0.026 & 0.027 & 0.5 & 0.018 & 0.019 & 0.020 & 0.022 & 0.024 & 0.026 & 0.027 \\
\hline 0.6 & 0.021 & 0.022 & 0.024 & 0.027 & 0.029 & 0.031 & 0.032 & 0.6 & 0.021 & 0.022 & 0.024 & 0.027 & 0.029 & 0.031 & 0.032 \\
\hline 0.7 & 0.025 & 0.026 & 0.028 & 0.031 & 0.035 & 0.036 & 0.038 & 0.7 & 0.025 & 0.026 & 0.028 & 0.031 & 0.035 & 0.036 & 0.038 \\
\hline 0.8 & 0.029 & 0.030 & 0.032 & 0.036 & 0.040 & 0.042 & 0.043 & 0.8 & 0.029 & 0.030 & 0.032 & 0.036 & 0.040 & 0.042 & 0.043 \\
\hline 0.9 & 0.033 & 0.034 & 0.037 & 0.041 & 0.045 & 0.047 & 0.049 & 0.9 & 0.033 & 0.034 & 0.037 & 0.041 & 0.045 & 0.047 & 0.049 \\
\hline 1.0 & 0.036 & 0.038 & 0.041 & 0.046 & 0.050 & 0.053 & 0.055 & 1.0 & 0.036 & 0.038 & 0.041 & 0.046 & 0.050 & 0.053 & 0.055 \\
\hline
\end{tabular}


TABLE 3

Continued.

\begin{tabular}{|c|c|c|c|c|c|c|c|c|c|c|c|c|c|c|c|}
\hline \multicolumn{4}{|c|}{ Annuity } & \multicolumn{4}{|c|}{$\gamma$} & \multirow[b]{2}{*}{$\epsilon$} & \multicolumn{7}{|c|}{$\gamma$} \\
\hline$\epsilon$ & $0 \%$ & $10 \%$ & $25 \%$ & $50 \%$ & $75 \%$ & $90 \%$ & $100 \%$ & & $0 \%$ & $10 \%$ & $25 \%$ & $50 \%$ & $75 \%$ & $90 \%$ & $100 \%$ \\
\hline 0.1 & 0.507 & 0.512 & 0.519 & 0.524 & 5.522 & 0.516 & 0.510 & 0.1 & 0.507 & 0.507 & 0.508 & 0.509 & 0.509 & 0.510 & 0.510 \\
\hline 0.2 & 1.068 & 1.080 & 1.094 & 1.108 & 1.105 & 1.093 & 1.079 & 0.2 & 1.068 & 1.069 & 1.071 & 1.073 & 1.076 & 1.078 & 1.079 \\
\hline 0.3 & 1.695 & 1.715 & 1.740 & 1.754 & 1.763 & 1.744 & 1.721 & 0.3 & 1.695 & 1.698 & 1.701 & 1.708 & 1.714 & 1.718 & 1.721 \\
\hline 0.4 & 2.402 & 2.432 & 2.047 & 2.512 & 2.516 & 2.492 & 2.456 & 0.4 & 2.402 & 2.407 & 2.415 & 2.429 & 2.443 & 2.451 & 2.456 \\
\hline 0.5 & 3.208 & 3.252 & 3.309 & 3.375 & 3.391 & 3.364 & 3.316 & 0.5 & 3.208 & 3.219 & 3.235 & 3.262 & 3.289 & 3.305 & 3.316 \\
\hline 0.6 & 4.139 & 4.201 & 4.284 & 4.387 & 4.428 & 4.403 & 4.343 & 0.6 & 4.139 & 4.160 & 4.190 & 4.241 & 4.292 & 4.323 & 4.343 \\
\hline 0.7 & 5.230 & 5.317 & 5.435 & 5.594 & 5.682 & 5.673 & 5.609 & 0.7 & 5.230 & 5.268 & 5.325 & 5.420 & 5.514 & 5.571 & 5.609 \\
\hline 0.8 & 6.528 & 6.648 & 6.818 & 7.063 & 7.236 & 7.272 & 7.224 & 0.8 & 6.528 & 6.597 & 6.702 & 6.876 & 7.050 & 7.155 & 7.224 \\
\hline 0.9 & 8.095 & 8.263 & 8.507 & 8.887 & 9.211 & 9.350 & 9.377 & 0.9 & 8.095 & 8.223 & 8.416 & 8.736 & 9.056 & 9.248 & 9.377 \\
\hline 1.0 & 10.021 & 10.257 & 10.611 & 11.202 & 11.793 & 12.147 & 12.384 & 1.0 & 10.021 & 10.257 & 10.611 & 11.202 & 11.793 & 12.147 & 12.384 \\
\hline
\end{tabular}


TABLE 4

RELATIVE DIFFERENCE WHEN COMPARING UNISEX VS. WEIGHTED APPROACH FOR SCR, FOR VARYING PROPORTION $(\gamma)$ MALE/FEMALE AND SHOCK $(\epsilon)$.

\begin{tabular}{|c|c|c|c|c|c|c|c|}
\hline \multicolumn{4}{|c|}{ Pure-endowment 20 years } & \multicolumn{4}{|c|}{$\gamma$} \\
\hline$\epsilon$ & $0 \%$ & $10 \%$ & $25 \%$ & $50 \%$ & $75 \%$ & $90 \%$ & $100 \%$ \\
\hline 0.1 & $0.00 \%$ & $0.04 \%$ & $0.08 \%$ & $0.09 \%$ & $0.06 \%$ & $0.03 \%$ & $0.00 \%$ \\
\hline 0.2 & $0.00 \%$ & $0.04 \%$ & $0.07 \%$ & $0.08 \%$ & $0.06 \%$ & $0.03 \%$ & $0.00 \%$ \\
\hline 0.3 & $0.00 \%$ & $0.03 \%$ & $0.06 \%$ & $0.07 \%$ & $0.05 \%$ & $0.02 \%$ & $0.00 \%$ \\
\hline 0.4 & $0.00 \%$ & $0.03 \%$ & $0.05 \%$ & $0.06 \%$ & $0.04 \%$ & $0.02 \%$ & $0.00 \%$ \\
\hline 0.5 & $0.00 \%$ & $0.02 \%$ & $0.04 \%$ & $0.05 \%$ & $0.04 \%$ & $0.02 \%$ & $0.00 \%$ \\
\hline 0.6 & $0.00 \%$ & $0.02 \%$ & $0.03 \%$ & $0.04 \%$ & $0.03 \%$ & $0.01 \%$ & $0.00 \%$ \\
\hline 0.7 & $0.00 \%$ & $0.01 \%$ & $0.03 \%$ & $0.03 \%$ & $0.02 \%$ & $0.01 \%$ & $0.00 \%$ \\
\hline 0.8 & $0.00 \%$ & $0.01 \%$ & $0.02 \%$ & $0.02 \%$ & $0.01 \%$ & $0.01 \%$ & $0.00 \%$ \\
\hline 0.9 & $0.00 \%$ & $0.00 \%$ & $0.01 \%$ & $0.01 \%$ & $0.01 \%$ & $0.00 \%$ & $0.00 \%$ \\
\hline 1.0 & $0.00 \%$ & $0.00 \%$ & $0.00 \%$ & $0.00 \%$ & $0.00 \%$ & $0.00 \%$ & $0.00 \%$ \\
\hline \multicolumn{4}{|c|}{ Pure-endowment 30 years } & \multicolumn{4}{|c|}{$\gamma$} \\
\hline$\epsilon$ & $0 \%$ & $10 \%$ & $25 \%$ & $50 \%$ & $75 \%$ & $90 \%$ & $100 \%$ \\
\hline 0.1 & $0.00 \%$ & $0.11 \%$ & $0.21 \%$ & $0.25 \%$ & $0.18 \%$ & $0.08 \%$ & $0.00 \%$ \\
\hline 0.2 & $0.00 \%$ & $0.10 \%$ & $0.19 \%$ & $0.23 \%$ & $0.16 \%$ & $0.07 \%$ & $0.00 \%$ \\
\hline 0.3 & $0.00 \%$ & $0.08 \%$ & $0.17 \%$ & $0.20 \%$ & $0.14 \%$ & $0.06 \%$ & $0.00 \%$ \\
\hline 0.4 & $0.00 \%$ & $0.07 \%$ & $0.14 \%$ & $0.17 \%$ & $0.12 \%$ & $0.05 \%$ & $0.00 \%$ \\
\hline 0.5 & $0.00 \%$ & $0.06 \%$ & $0.12 \%$ & $0.14 \%$ & $0.10 \%$ & $0.05 \%$ & $0.00 \%$ \\
\hline 0.6 & $0.00 \%$ & $0.05 \%$ & $0.10 \%$ & $0.12 \%$ & $0.08 \%$ & $0.04 \%$ & $0.00 \%$ \\
\hline 0.7 & $0.00 \%$ & $0.04 \%$ & $0.07 \%$ & $0.09 \%$ & $0.06 \%$ & $0.03 \%$ & $0.00 \%$ \\
\hline 0.8 & $0.00 \%$ & $0.03 \%$ & $0.05 \%$ & $0.06 \%$ & $0.04 \%$ & $0.02 \%$ & $0.00 \%$ \\
\hline 0.9 & $0.00 \%$ & $0.01 \%$ & $0.02 \%$ & $0.03 \%$ & $0.02 \%$ & $0.01 \%$ & $0.00 \%$ \\
\hline 1.0 & $0.00 \%$ & $0.00 \%$ & $0.00 \%$ & $0.00 \%$ & $0.00 \%$ & $0.00 \%$ & $0.00 \%$ \\
\hline \multicolumn{4}{|c|}{ Annuity } & \multicolumn{4}{|c|}{$\gamma$} \\
\hline$\epsilon$ & $0 \%$ & $10 \%$ & $25 \%$ & $50 \%$ & $75 \%$ & $90 \%$ & $100 \%$ \\
\hline 0.1 & $0.00 \%$ & $0.98 \%$ & $2.09 \%$ & $2.98 \%$ & $2.48 \%$ & $1.31 \%$ & $0.00 \%$ \\
\hline 0.2 & $0.00 \%$ & $1.00 \%$ & $2.15 \%$ & $3.09 \%$ & $2.62 \%$ & $1.41 \%$ & $0.00 \%$ \\
\hline 0.3 & $0.00 \%$ & $1.01 \%$ & $2.19 \%$ & $3.21 \%$ & $2.77 \%$ & $1.52 \%$ & $0.00 \%$ \\
\hline 0.4 & $0.00 \%$ & $1.02 \%$ & $2.23 \%$ & $3.30 \%$ & $2.91 \%$ & $1.63 \%$ & $0.00 \%$ \\
\hline 0.5 & $0.00 \%$ & $1.02 \%$ & $2.23 \%$ & $3.35 \%$ & $3.03 \%$ & $1.74 \%$ & $0.00 \%$ \\
\hline 0.6 & $0.00 \%$ & $0.99 \%$ & $2.18 \%$ & $3.32 \%$ & $3.07 \%$ & $1.82 \%$ & $0.00 \%$ \\
\hline 0.7 & $0.00 \%$ & $0.91 \%$ & $2.03 \%$ & $3.12 \%$ & $2.96 \%$ & $1.80 \%$ & $0.00 \%$ \\
\hline 0.8 & $0.00 \%$ & $0.76 \%$ & $1.70 \%$ & $2.65 \%$ & $2.57 \%$ & $1.61 \%$ & $0.00 \%$ \\
\hline 0.9 & $0.00 \%$ & $0.48 \%$ & $1.08 \%$ & $1.70 \%$ & $1.68 \%$ & $1.08 \%$ & $0.00 \%$ \\
\hline 1.0 & $0.00 \%$ & $0.00 \%$ & $0.00 \%$ & $0.00 \%$ & $0.00 \%$ & $0.00 \%$ & $0.00 \%$ \\
\hline
\end{tabular}


From Tables 3 and 4, we can observe what follows:

1. As expected from Proposition 3.1 and Corollary 3.2, for all life insurance products, all $\epsilon$ and all $\gamma, S C R^{u}$ is greater or equal than $S C R^{\text {weighted }}$. When $\epsilon$ is 0 or 1 , and when $\gamma$ is 0 or 1 , there is no difference between $S C R^{u}$ and $S C R^{\text {weighted }}$, and their gap is 0 . For the pure endowment products, in all cases the differences are smaller than 0.001 so they cannot be appreciated from Table 3. However, the difference is non-negligible for the annuity product.

2. For all $\epsilon$ and $\gamma$ both the absolute gap $S C R^{u}-S C R^{\text {weighted }}$ and the relative gap ( $\left.S C R^{u}-S C R^{\text {weighted }}\right) / S C R^{u}$ are lowest for pure endowment 20 years, slightly higher for pure endowment 30 years, highest for the annuity. In all cases, the relative gap is bigger than the absolute gap because the denominator $S C R^{u}$ is lower than one. The order of magnitude of the relative gap is negligible for the pure endowment for both durations, reaching a maximum of $0.25 \%$ for pure endowment with 30 years, with $\epsilon=0.1$ and $\gamma=0.5$. The order of magnitude of the relative gap is more important for the annuity case, reaching a maximum of $3.35 \%$ for $\epsilon=0.5$ and $\gamma=0.5$.

3. Dependence on $\gamma$ of $S C R^{u}$ and $S C R^{\text {weighted }}$. Interestingly, for all products, in most cases when $\epsilon$ is fixed, both $S C R^{u}$ and $S C R^{\text {weighted }}$ increase when $\gamma$ increases. This is apparently counterintuitive, if one thinks that $\gamma=0$ means a portfolio consisting of females and $\gamma=1$ means a portfolio consisting of males only. Because females are riskier than males for pure endowment and annuity products, one would expect a greater SCR for females than for males. But this can be explained observing that by definition the SCR is the difference between what insurers should pay in case of distorted higher survival probabilities and what insurers have already set aside in the reserves with the single premium, see (10). Indeed, let us consider, for simplicity the SCR at time 0 for the pure endowment case for gender $i$ :

$$
S C R_{\text {end }-T}^{i}(\epsilon)=e^{-r T}\left(S^{i}(T)^{1-\epsilon}-S(T)\right)=e^{-r T} S^{i}(T)^{1-\epsilon}-\Pi^{i},
$$

where $\Pi^{i}$ is the fair price for gender $i$. Obviously, the fact that $S^{m}(T)<$ $S^{f}(T)$ produces $S^{m}(T)^{1-\epsilon}<S^{f}(T)^{1-\epsilon}$, and therefore the amount to be paid in absolute terms with a distorted survival probability is higher for females than for males; however, in the SCR we have to subtract the fair premium $\Pi^{i}=e^{-r T} S^{i}(T)$ that for the females is higher than for the males. Thus, if the fair premium for females is higher than the fair premium for males, and if the latter is higher than 0.35 (which happens to be the case for pure endowment 20 or 30 years issued to 35-years old policyholder), then we have ${ }^{5}$

$$
S^{f}(T)^{1-\epsilon}-S^{f}(T)<S^{m}(T)^{1-\epsilon}-S^{m}(T) \Rightarrow S C R_{\text {end }-T}^{f}(\epsilon)<S C R_{\text {end }-T}^{m}(\epsilon) .
$$


In all intermediate situations, when $\gamma \in(0,1)$ we have

$$
S C R_{\text {end }-T}^{f}(\epsilon)<\gamma S C R_{\text {end }-T}^{m}(\epsilon)+(1-\gamma) S C R_{\text {end }-T}^{m}(\epsilon)<S C R_{\text {end }-T}^{m}(\epsilon),
$$

that explains the increasing $S C R$ with $\gamma$, both for the unisex case and the weighted case. This explanation holds for the pure endowment only, and in fact for the annuity in some cases $S C R^{u}$ does not increase with $\gamma$.

4. Dependence on $\gamma$ of absolute and relative gap of $S C R^{u}$ and $S C R^{\text {weighted }}$. When $\epsilon$ is fixed, the maximum relative gap is with $\gamma=0.5$ for all products considered. This is consistent with intuition: the most unfair situation is when the portfolio is perfectly balanced in terms of males and females. As a degenerate case, when there is only one gender in the portfolio, the unisex price is the gender-based fair price, and the EU Gender Directive does not impact the price and the SCR. When there is a majority of one gender, the unisex price is strongly correlated with the gender-based fair price. The worst situation is when there is the same number of males and females.

5. Dependence on $\epsilon$. When $\gamma$ is fixed, both $S C R^{u}$ and $S C R^{\text {weighted }}$ increase with $\epsilon$, for all products. This is due to the fact that a greater shock to the survival probabilities produces a higher SCR. This has different impact on the absolute and relative gap between $S C R^{u}$ and $S C R^{\text {weighted }}$. For the pure endowment with both durations, the absolute gap remains almost stable with $\epsilon$, and the relative gap decreases with $\epsilon$, the maximum being at $\epsilon=0.1$. For the annuity both the absolute and relative gap increase with $\epsilon$.

\section{IMPLICATIONS OF POSSIBLE ALTERNATIVE PRACTICES ON UNISEX SCR}

\subsection{Two possible ways to deal with the EU Gender Directive}

Insurance companies do not always seem to adopt the unisex fairness principle in pricing unisex policies. Although officially there is no clear disclosure of pricing procedures, in practice there seem to be two ways to do unisex pricing: prevailing risk and weighted risk.

6.1.1. Prevailing risk: "max-risk procedure". A possible way to deal with the EU Gender Directive is to consider the mixed portfolio as if it were made only by high-risk policyholders, such as females for the pure endowment or the annuity, and males for the term insurance or the whole life insurance. We are going to call this procedure "max-risk-procedure": it is very conservative and implies charging always the maximum between the two gender-based prices. It is obvious that the price charged with the max-risk-procedure is higher than the unisex fair premium, and, in the considered cases of pure endowment and lifetime annuity, their difference increases with the males' portfolio share $\gamma$. 
6.1.2. Weighted risk: "weight-load procedure". Another possible way to deal with the EU Gender Directive is to take all the 1-year survival rate of males and females and to mix them with weights that reflect both the portfolio composition $\gamma$ and the product issued, with an additional loading $\eta$ on females or males, depending on whether the product covers the risk of survival or the risk of death. We are going to call this procedure "weight-load procedure". In particular, when the product covers against the risk of survival (pure endowment, annuity), more weight is given to the females survival rate and the unisex 1-year survival rate is given by

$$
p_{x}^{w l}=(\gamma-\eta) p_{x}^{m}+(1-(\gamma-\eta)) p_{x}^{f}
$$

for some $0 \leq \eta \leq \gamma \cdot{ }^{6}$ We stress that the weight-load-procedure is not equivalent to the unisex fairness principle procedure, even with $\eta=0$. Indeed, the unisex fairness principle implies that for the pure endowment duration $T$ the $T$-year unisex survival probability is a weighted average with weights $\gamma$ and $1-\gamma$ of the males' and females' $T$-year survival probabilities (see (7)), while for the annuity the $k$-year unisex survival probabilities are weighted averages with weights $\gamma$ and $1-\gamma$ of the males' and females' $k$-years survival probabilities for all $k=1, \ldots \omega-x$ (see (9)). It is not difficult to see that these conditions are violated if the survival rates satisfy (37), also with $\eta=0$. Therefore, the unisex price charged with the weight-load procedure is different from the unisex fair premium, and in the practice it turns out to be generally higher than that.

\subsection{Consequences for the calculation of the SCR}

In this section, we compare between the SCR calculated with the two alternative pricing procedures illustrated in Section 6.1 and the fair SCR, SCR $R^{\text {weighted }}$ calculated in Section 5.2.

We fix an equal proportion of genders in the portfolio, $\gamma=0.5$, and a shock on the survival probabilities $\epsilon=0.5$, and analyze the three products considered in Section 5, namely the pure endowment 20 years, the pure endowment 30 years and the annuity. For the weight-load procedure we set $\eta=0.1,0.3,0.5$. Therefore, due to (37), the results for the max-risk procedure coincide with those of the weight-load procedure in the case $\eta=0.5$.

Table 5 reports the $S C R$ with the weight-load procedure, $S C R^{w l}$, and the $S C R$ for the max-risk procedure, $S C R^{m r}$, that coincides with $S C R^{w l}$ with $\eta=$ 0.5 . It also reports their absolute gap with respect to $S C R^{\text {weighted }}$ and their relative gap, for instance, $\left(S C R^{m r}-S C R^{\text {weighted }}\right) / S C R^{m r}$.

We observe that the SCR for the max-risk procedure is always lower than the $S C R^{\text {weighted }}$. The explanation is equal to that given in comment 3 from Section 5.2 to explain why the SCR for females is lower than that for males. If the insurance company behaves as if there are only females in the mixed portfolio, the price charged to the males of the portfolio is higher than what should be, so less money is needed for the SCR, because excessive money has been set aside by the insurance company. This is certainly a safe procedure for the solvency of the company, but the price of this cautious procedure is paid only by the customers. 
TABLE 5

$S C R_{z}^{w l}(\epsilon)$ AND $S C R^{m r}(\epsilon)$ : DIFFERENCE AND RELATIVE DIFFERENCE TO $S C R^{\text {weighted }}(\epsilon)$ FOR $\epsilon=0.5$ AND $\gamma=0.5$ DEPENDING ON $\eta$ AND TYPE OF PRODUCT. $S C R^{m r}(\epsilon)$ IS THE CASE $S C R^{w l}(\epsilon)$ WITH $\eta=0.5$.

\begin{tabular}{|c|c|c|c|}
\hline \multicolumn{4}{|c|}{ Pure-endowment 20 years $\left(S C R^{\text {weighted }}=0.012\right)$} \\
\hline & \multicolumn{3}{|c|}{$\eta$} \\
\hline & 0.1 & 0.3 & 0.5 \\
\hline$S C R^{w l}$ & 0.012 & 0.011 & 0.010 \\
\hline$S C R^{w l}-S C R^{\text {weighted }}$ & 0.000 & -0.001 & -0.003 \\
\hline Relative diff. & $-4.08 \%$ & $-13.74 \%$ & $-25.46 \%$ \\
\hline \multicolumn{4}{|c|}{ Pure-endowment 30 years $\left(S C R^{\text {weighted }}=0.022\right)$} \\
\hline & \multicolumn{3}{|c|}{$\eta$} \\
\hline & 0.1 & 0.3 & 0.5 \\
\hline $\operatorname{SCR}_{z}^{w l}(\epsilon)$ & 0.021 & 0.020 & 0.018 \\
\hline$S C R^{w l}-S C R^{\text {weighted }}$ & -0.001 & -0.003 & -0.004 \\
\hline Relative diff. & $-3.72 \%$ & $-13.17 \%$ & $-24.71 \%$ \\
\hline \multicolumn{4}{|c|}{ Annuity $\left(S C R^{\text {weighted }}=3.268\right)$} \\
\hline & \multicolumn{3}{|c|}{$\eta$} \\
\hline & 0.1 & 0.3 & 0.5 \\
\hline$S C R^{w l}$ & 3.285 & 3.259 & 3.218 \\
\hline$S C R^{w l}-S C R^{\text {weighted }}$ & 0.017 & -0.009 & -0.050 \\
\hline Relative diff. & $0.52 \%$ & $-0.28 \%$ & $-1.55 \%$ \\
\hline
\end{tabular}

The weight-load procedure gives an intermediate situation between the maxrisk procedure and the weighted procedure. This is due to the fact that the survival probabilities used are not equal to the females' ones as in the max-risk procedure, but they are closer to the females' ones than they should be with just volume-related weights, due to the extra loading $\eta$.

We notice a remarkable difference between the pure endowment and the annuity. For both pure endowments, the difference $S C R^{w l}-S C R^{\text {weighted }}$ is always lower than 0 and the relative difference becomes as high as $-25 \%$ for higher values of $\eta$. For the annuity, $S C R^{w l}-S C R^{\text {weighted }}$ is positive with low values of $\eta$ and becomes negative when $\eta$ increases, but the relative difference is low. This means that there is an intermediate value of $\eta$ for which the weight-load procedure equals the weighted pre-Gender Directive approach. 


\section{DISCUSSION AND CONCLUSION}

Starting from the evidence that the EU Gender Directive obliges issuers of life insurance products to charge the same premium to policyholders of different genders, we address the issue of calculating SCRs for pure endowments and annuities issued to mixed portfolios. We strongly support the use of the unisex fairness principle in the calculation of the unisex single premium. We analyze the SCR in the following two possible situations: (i) using the unisex fairness principle; (ii) not using it.

(i) Assuming that the insurer charges the unisex fair premium, we notice that he can calculate the SCR in two different ways:

1. first, with the unisex fair survival probability, as if the mixed portfolio was made by $m+n$ homogeneous mixed policyholders with fair unisex survival probabilities; with this procedure we calculate the quantities $S C R^{u}$;

2. second, mixing the SCR of males and females with volume-related weights, as if the mixed portfolio was made by two subportfolios of $m$ males and $n$ females (in the same way it was done before the EU Gender Directive, see Remark 2); with this procedure we calculate the quantities $S C R^{\text {weighted }}$.

We notice that the fair premium charged to the policyholders is the same in the two cases. In Section 3.2, we show that the SCR at issuing time calculated with the fair unisex survival probabilities is higher than or equal to that calculated for the two subportfolios. In other words, if insurers treat the mixed portfolio as a portfolio of homogeneous unisex policyholders with a fair unisex survival probability, they set aside for solvency requirements more money than they would have done before the EU Gender Directive. We find that the relative gap between the SCRs calculated in the two different ways is negligible for pure endowments, and it is at most of the order of $3 \%$ for annuities. The unisex SCR, $S C R^{u}$, can be considered a good approximation of the fair $S C R^{\text {weighted }}$, for pure endowments, and a cautious SCR for annuities.

(ii) Assuming that the insurer does not use the unisex fairness principle to calculate the unisex tariff, we find that it is common practice to use survival probabilities closer or equal to those of the females for pure endowments and annuities. We calculate the SCR according to the distorted survival probabilities adopted. We get different results for pure endowments and annuities. For pure endowments, in all cases, the distorted SCR is lower than the $S C R^{\text {weighted }}$ that would have been calculated before the EU Gender Directive. The relative difference can be as high as $-25 \%$. The reason of this apparently counterintuitive inequality lies in the fact that with distorted survival probabilities the premium charged by insurers is much higher than the unisex fair price. Therefore, more money than needed is set aside in the reserves at the policy inception, and less money than needed is required for the SCR. Both a higher unisex tariff and a lower SCR seem good news for the insurer. However, the price to be paid for this advantage is paid entirely by the customer, who pays more than needed for the policy. For the annuity the SCR calculated with distorted survival 
probabilities is lower than the $S C R^{\text {weighted }}$ if enough extra loading is assigned to the females' survival probabilities, while it becomes higher than that for low enough extra-loading on females' survival probabilities. A correct selection of the extra-loading becomes then relevant to insurers.

We consider the present paper as the starting point of a more articulate research project on the effects of the EU Gender Directive on the calculation of SCRs for life insurance products. Products that pay in case of death such as whole and term life insurance are missing from the present analysis but are important too. A detailed analysis of the calculation of the SCR for products that pay in case of death is subject of ongoing research.

\section{ACKNOWLEDGEMENTS}

We are indebted to Kristian Buchardt and Paolo De Angelis for very valuable comments that improved the paper. We thank Spanish Ministry Grant/FEDER ECO2016-76203-C2-2-P.

\section{NOTES}

1. In the remainder of the paper, we will refer to the EU Gender Directive; however, the results apply to similar no-gender discrimination rules anywhere in the world. Indeed, the analysis of gender equalization is relevant not only to the European context. In 1978, the United States Supreme Court first prohibited gender-based divisions in insurance in the case City of Los Angeles. In 1983, the courts banned gender-based insurance distinctions for Tax Deferred Annuity and Deferred Compensation Plans in Arizona. Insurance companies have opposed any legislation that restrict their ability to use gender-based distinctions in developing insurance classifications, rates and coverages, but fighting discrimination is on the agenda of social movements all over the world.

2. This product is similar to immediate lifetime annuity product in Milevsky and Salisbury (2015). Unlike Milevsky and Salisbury (2015) where the optimal annuity payoff is determined, we are more interested in the SCR related to these products.

3. In some countries like Denmark insurers receive fiscal incentives if they merge the two subportfolios.

4. Chen and Vigna (2017) make sensitivity analysis with respect to $\rho$ and find that results are almost insensitive to changes in $\rho$.

5. From the mathematical point of view, this can be explained as follows. If $\alpha \in(0,1)$ the function $g(x)=x^{\alpha}-x$ is decreasing for $x \in\left(\alpha^{1 /(1-\alpha)}, 1\right)$. We notice that when $\alpha$ ranges in $(0,1)$, $\alpha^{1 /(1-\alpha)}$ ranges between 0.08 and 0.35 . Given the initial age 35 and the duration $T=20,30$ years, we then have $\alpha^{1 /(1-\alpha)}<S^{m}(T)<S^{f}(T)$, that implies $g\left(S^{m}(T)\right)>g\left(S^{f}(T)\right)$ in all cases of pure endowment.

6. When the product covers against the risk of death (term insurance, whole life), more weight is given to the males survival rate and the unisex 1-year survival rate is given by $p_{x}^{w l}=(\gamma+\eta) p_{x}^{m}+$ $(1-(\gamma+\eta)) p_{x}^{f}$

\section{REFERENCES}

Aseervatham, V., LeX, C. and Spindler, M. (2016) How do unisex rating regulations affect gender differences in insurance premiums? The Geneva Papers on Risk and Insurance Issues and Practice, 41(1), 128-160. 
BIFFIS, E. (2005) Affine processes for dynamic mortality and actuarial valuations. Insurance: Mathematics and Economics, 37(3), 443-468.

BöRGER, M. (2010) Deterministic shock vs. stochastic value-at-risk, an analysis of the solvency II standard model approach to longevity risk. Blätter der DGVFM, 31(2), 225-259.

ChEn, A. and VignA, E. (2017) A unisex stochastic mortality model to comply with EU Gender Directive. Insurance: Mathematics and Economics, 73, 124-136.

DAHL, M. (2004) Stochastic mortality in life insurance: Market reserves and mortality-linked insurance contracts. Insurance: Mathematics and Economics, 35(1), 113-136.

Duffie, D., PAn, J. and Singleton, K. (2000) Transform analysis and asset pricing for affine jump-diffusions. Econometrica, 68(6), 1343-1376.

EIOPA (2014). Technical specifications for the Solvency II Preparatory Phase - Part I. Technical report, European Insurance and Occupational Pensions Authority.

Guillen, M. (2012) Sexless and beautiful data: From quantity to quality. Annals of Actuarial Science, 6(02), 231-234.

LIN, Y. and COX, S. H. (2005) Securitization of mortality risks in life annuities. Journal of Risk and Insurance, 72(2), 227-252.

LuCiAno, E. and Vigna, E. (2008) Mortality risk via affine stochastic intensities: Calibration and empirical relevance. Belgian Actuarial Bulletin, 8(1), 5-16.

Milevsky, M. A. and Promislow, S. D. (2001) Mortality derivatives and the option to annuitise. Insurance: Mathematics and Economics, 29(3), 299-318.

MileVsKY, M. A. and SAlisbuRY, T. S. (2015) Optimal retirement income tontines. Insurance: Mathematics and Economics, 64, 91-105.

OliVIERI, A. and PitACCO, E. (2009) Stochastic mortality: The impact on target capital. ASTIN Bulletin, 39(02), 541-563.

ORNELAS, A. and GUILLEN, M. (2013) A comparison between general population mortality and life tables for insurance in Mexico under gender proportion inequality. Revista de Métodos Cuantitativos para la Economía y la Empresa. Journal of Quantitative Methods for Economics and Business Administration, 16(1), 47-67.

SASS, J. and SEIFRIED, F. T. (2014) Insurance markets and unisex tariffs: Is the European Court of Justice improving or destroying welfare? Scandinavian Actuarial Journal, 2014(3), 228254.

SCHMEISER, H., StÖRMER, T. and WAGNER, J. (2014) Unisex insurance pricing: Consumers' perception and market implications. The Geneva Papers on Risk and Insurance Issues and Practice, 39(2), 322-350.

ThierY, Y. and VAN SCHOUBroecK, C. (2006) Fairness and equality in insurance classification. The Geneva Papers on Risk and Insurance Issues and Practice, 31(2), 190-211.

University of CAlifornia, Berkeley (USA) and MAX Planck Institute FOR DeMOgRAPhic Research (Germany) (2002) Human Mortality Database. Available at www.mortality.org or www.humanmortality.de.

\author{
AN CHEN \\ Faculty of Mathematics and Economics \\ University of Ulm, Helmholtzstrasse 20 \\ 89069 Ulm, Germany \\ E-Mail:an.chen@uni-ulm.de
}

MontserRat Guillen

Department of Econometrics

University of Barcelona

Riskcenter-IREA, Av. Diagonal 690

08034 Barcelona, Spain

E-mail:mguillen@ub.edu 
ELENA VignA (Corresponding author)

University of Torino

Collegio Carlo Alberto and CeRP

Corso Unione Sovietica 218 bis

10134 Torino, Italy

E-Mail: elena.vigna@unito.it 